\title{
Heidegger et Deleuze : même combat?
}

\author{
Pierre-Alexandre Fradet $_{\text {t }}$
}

$\mathrm{Au}$ premier abord, voire peut-être aussi au second, tout semble opposer Heidegger à Deleuze. Alors que l'Allemand nous invite à prendre expressément conscience de la mort, phénomène imminent, incontournable et qui ne laisse pas d'éveiller l'angoisse, le Français nous dispense de la tâche de méditer à toute heure cette mort ( $c f$. Adkins). Tandis que Heidegger fait « de Nietzsche le plus grand et le dernier penseur de la métaphysique », Deleuze voit volontiers «dans le nietzschéisme une philosophie qui remplace la métaphysique par un autre mode de pensée » (Sergeant 15; cf. aussi Winchester ch. 2-3 et Vaysse). Heidegger constate le primat actuel de la technique et le dépeint comme un «péril » (Heidegger, « Technique » 43); Deleuze, pour sa part, emploie le concept de «machines de guerre» pour définir les forces « irréductible[s] à l'appareil d'État » (Deleuze et Guattari, Plateaux 435), le langage de la technique lui permettant ici de désigner ce qu'il y a de louable à ses yeux ${ }^{1}$. Autre raison de voir entre Heidegger et Deleuze un fossé plutôt qu'un pont : «les avancées les plus décisives de la pensée deleuzienne », comme le remarque Alain Beaulieu, «sont décidées dans un corps à corps énergique, virulent, contigu et soutenu avec la doctrine phénoménologique, son amie-ennemie de toujours »(Beaulieu, Deleuze 260).

C'est que Deleuze se fait un point d'honneur de définir sa philosophie par opposition à celle de Heidegger. Ainsi, là où l'Allemand s'efforce d'interpréter le sens de l'être, le Français 
«s'approprie l'énoncé heideggérien suivant lequel la pensée ne pense pas encore, le détourne de son sens originel $[\ldots]$ et il fait de la pensée véritable une pensée présente et traversée par des grandeurs qui l'amènent à expérimenter la puissance de l'affirmation ${ }^{2}$ » (43, nous soulignons). Où donc faire converger Heidegger et Deleuze? Un rapprochement minimal peut-il être établi? Certains estiment que oui. En tête de liste, on mentionnera Benoît Goetz qui a repéré une filiation entre Heidegger et Deleuze sur la question du « devenir-animal » (cf. aussi les travaux de Pieron, Buchanan, Memon, Brown et Beaulieu, «Status of Animality »). Mais les terrains d'entente ne sont pas légion entre les deux auteurs, et la question se pose avec intérêt : outre sur le thème du devenir-animal, Heidegger et Deleuze peuvent-ils fraterniser et ainsi se prêter un mutuel appui?

Cette question nous occupera dans ce qui suit. Nous y répondrons par l'affirmative, mais avec moult nuances, en trois temps. Il s'agira d'abord de montrer que les pensées heideggérienne et deleuzienne se recoupent par leur volonté de mettre à bas les acceptions classiques du possible. Nous ferons voir, dans un second temps, que Heidegger et Deleuze se rejoignent aussi par leurs manières respectives de repenser le problème du possible. En troisième lieu, plongeant dans les enjeux pratiques, nous nous demanderons si le projet deleuzien n'est pas susceptible de compléter la philosophie heideggérienne. Notre objectif ne sera donc pas d'effacer de manière obtuse la distance entre Heidegger et Deleuze; il consistera, bien plutôt, à atténuer l'important écart qu'on voit d'ordinaire entre eux, et tout à la fois à faire signe vers une certaine complémentarité, encore inaperçue jusqu'ici. 


\section{Un moment négatif : mise en question des acceptions classiques du possible}

C'est un fait courant de repérer dans l'histoire de la philosophie un geste de subordination du possible au réel. Dans sa Métaphysique, Aristote affirme par exemple que l'acte a préséance sur la puissance sous quatre angles principaux $(\Theta, 8 ; c f$. aussi Witt). Dans l'Encyclopédie des sciences philosophiques (396) et dans la Science de la logique (199 et 204), Hegel suggère quant à lui que toute possibilité, pour être digne de ce nom, doit s'actualiser un jour ou l'autre. Portant leur regard sur ces conceptions du possible et sur celles qui en sont issues, Heidegger et Deleuze éprouveront un vif sentiment d'insatisfaction. L'un et l'autre s'accorderont donc à dire qu'il faut revoir les alternatives qu'offre la métaphysique pour penser l'ordre modal. Avant d'expliciter leur propre réponse à la question du possible, ils procèdent négativement : ils disent en quoi ne consiste pas leur concept de possibilité. Tirer au clair ce versant négatif permettra de dégager un souci commun aux deux auteurs, celui de se distancier de la tradition métaphysique.

Considérons d'abord la pensée heideggérienne. Dans le paragraphe 31 d'Etre et temps, Heidegger distingue la «possibilité existentiale », qu'il cherche à mettre en valeur, d'un ensemble d'acceptions courantes. On peut repérer ici une quadruple négation. Tout d'abord, la possibilité existentiale ne correspond pas à (i) la «possibilité logique » ou à (ii) un « pouvoir-être flottant ». La première se dit de tout ce qui n'est ni inimaginable ni contradictoire en soi, et le second se comprend comme une certaine indifférence aux choix, décisions et engagements. $\mathrm{Si}$ aucun de ces concepts n'est adéquat selon Heidegger, c'est que les deux passent sous silence le fait que le Dasein, siège de la possibilité existentiale, se trouve en position d'«être-jeté » (Heidegger, Être et temps 127), c'est-à-dire sans cesse engagé dans des possibilités déterminées, 
concrètes. Mais le concept heideggérien de possible ne s'apparente pas davantage à (iii) la « contingence ». En réduisant la possibilité à celle-ci, on assumerait le langage métaphysique qui fait de la contingence une propriété accidentelle et l'on perdrait de vue le caractère essentiel du possible pur. C'est que l'idée de contingence renvoie à une sphère étrangère à la nécessité, au lieu que la possibilité existentiale, lorsque bien comprise, implique un caractère incontournable (127).

À ces trois négations, Heidegger en ajoute une autre : il distingue son concept de possible de (iv) la «possibilité réelle » et « existentielle » (128), autrement dit ce qui est réalisable, ou réalisé, dans le cadre des circonstances existantes. Le philosophe reconnaît certes que le Dasein s'engage toujours dans le concret et qu'en tout temps, en tout lieu, « il s'est à chaque fois déjà projeté » (128); mais il refuse d'identifier trait pour trait la possibilité existentiale à ce sens du possible. Car cette identification serait par trop réductrice: elle suggèrerait une similitude parfaite entre deux concepts inassimilables — les possibilités réelles et la structure même qui gère, organise, conditionne ces possibilités. «[L]e Dasein est constamment "plus” qu'il n'est factuellement », écrit Heidegger; «[e]n revanche, il n'est jamais plus qu'il n'est facticement » (128). On conviendra donc que toutes les possibilités réelles sont débordées dans leur factualité par un pouvoir-être factice, de fonction structurelle et irréductible à une possibilité donnée.

Dans cette analyse du factuel et du factice, dans cet examen du pouvoir-être propre au Dasein, se fait jour l'idée de débordement. Or, un débordement, un excédent constant, Heidegger est d'avis qu'il en existe un au centre de l'expérience du Dasein; car ce dernier se projette toujours dans ce qu'il n'est pas (195). Que doit-on entendre par le concept d'excédent constant? Heidegger se pose lui-même la question. Pour y répondre, il passe en revue trois hypothèses et les écarte tour à tour. (i) Il suppose d'abord que l'excédent constant correspond à un « être en 
dette »: «Est en excédent, par exemple, le reste d'un règlement encore à percevoir. [...] La liquidation de la "dette" en tant que levée de l'excédent signifie la "rentrée", c'est-à-dire l'arrivée successive du reste, rentrée par laquelle le ne-pas-encore est pour ainsi dire comblé » (195). Puisque la notion de dette implique qu'un certain dû fasse défaut, c'est-à-dire ne soit pas encore acquitté, il y a bien lieu de caractériser la dette comme un excédent. Toujours est-il que ce sens du terme ne satisfait pas Heidegger, qui s'abstient de le considérer comme l'excédent constant. Le passage suivant l'explique bien : "L'étant où quelque chose est encore en excédent a le mode d'être de l'à-portée-de-la-main. [...] Cependant, [...] ce faire-défaut comme excédent ne saurait en aucun cas déterminer ontologiquement le ne-pas-encore qui appartient au Dasein » (195 sq.). Que l'être en dette soit un excédent qui pourrait cesser de faire défaut, alors que l'excédent constant représente autre chose qu'une réalité contingente, intramondaine, un surplus auquel on pourrait venir à bout, cela explique donc que Heidegger rejette sa première hypothèse.

(ii) Autre tentative, même résultat : le philosophe conjecture que l'excédent constant est assimilable au «dernier quartier de lune» (196). Cette hypothèse semble quitter le sol de la contingence et se rapprocher de la nécessité ontologique, car le dernier quartier, ni plus ni moins, appartient à la lune. Pourtant un problème persiste : tandis que le dernier quartier de lune se dérobe au regard tout en restant accessible (le côté obscur d'un objet physique peut toujours s'éclaircir), «le ne-pas-encore appartenant au Dasein [l'excédent constant] ne demeure pas seulement inaccessible provisoirement et temporairement à l'expérience propre et étrangère » (196).

(iii) Insatisfait de cette hypothèse, Heidegger en proposera donc une troisième, qui fait directement écho à l'idée de puissance chez Aristote. De même que le second sens d'excédent avait paru plus essentiel que le premier, le troisième semble gagner en pertinence par rapport au 
second : «Le fruit vert [...] se presse vers sa maturité. Ce qu'il n'est pas encore ne lui est alors nullement ajouté, dans la maturation, en tant que pas-encore-sous-la-main. C'est lui-même au contraire qui se porte à la maturité, et un tel se-porter caractérise son être en tant que fruit » (196). Le fruit mûrissant ressortit à un excédent : il rougit, s'accomplit, mûrit, coïncidant dans son devenir avec l'immaturité même (196). À la différence de ce fruit, toutefois, le Dasein n'a aucune essence à réaliser. Non pas qu'il n'évolue guère en se projetant dans ce qu'il n'est pas; mais son accomplissement diffère de celui associé au fruit. Porté à maturité, le fruit s'est accompli, il a épuisé ses possibilités. À l'opposé, au moment de sa mort, le Dasein parvient à une limite terminale, mais «il finit dans l'inaccomplissement, à moins qu'il ne soit défait ou usé » $(197)^{3}$.

La conclusion coule alors de source : ni le premier, ni le second, ni le troisième sens du concept d'excédent, où l'on constate une divergence entre la situation du fruit et celle du Dasein, porteur d'un excédent constant, ne permettent selon Heidegger de rendre compte de ce concept.

Deleuze se réclame-t-il davantage de la tradition lorsqu'il se penche sur l'ordre modal? Il s'en faut de beaucoup. À vrai dire, toute la pensée deleuzienne est suspendue à une critique radicale des acceptions classiques du possible. Cette critique s'inspire largement de la philosophie de Bergson (Deleuze, Différence 274; cf. aussi Bergsonisme 100 sq.) ${ }^{4}$ et, à bien des égards, elle s'oppose à celle de Leibniz (Différence 274 sq.). Si Deleuze évoque surtout l'idée de «possibilité logique » (274) et ne s'en prend pas de façon directe, dans Différence et répétition, à d'autres sens du possible, son analyse laisse croire qu'il veut fustiger l'ensemble des principaux sens que la tradition lui rattache. Une fois réduite à son essence, cette critique paraît s'appuyer sur trois arguments centraux ${ }^{5}$, dont le corollaire principal est l'introduction du concept de « virtuel ». 
1) Le premier d'entre eux a trait au rapport complexe entre le possible et le réel :

Le seul danger, [...] c'est de confondre le virtuel avec le possible. Car le possible s'oppose au réel; le processus du possible est donc une "réalisation». Le virtuel, au contraire, ne s'oppose pas au réel; il possède une pleine réalité par lui-même. Son processus est l'actualisation. On aurait tort de ne voir ici qu'une dispute de mots : il s'agit de l'existence elle-même. Chaque fois que nous posons le problème en termes de possible et de réel, nous sommes forcés de concevoir l'existence comme un surgissement brut, acte pur, saut qui s'opère toujours derrière notre dos, soumis à la loi du tout ou rien. (272sq.)

Pour emprunter une expression à Bergson, la possibilité n'est autre qu'une idéalité dont «l'acquisition d'existence »(Bergson, «Possible et réel » 112) reste encore à venir. Le possible est donc traditionnellement conçu, pour ainsi dire, comme étant moins que le réel. Cette acception de l'idée de possible est fort regrettable selon Deleuze qui endosse avec Bergson le présupposé suivant ${ }^{6}:$ tout concept, toute idée et tout geste sont forcément conçus ou faits à l'intérieur de la sphère du réel'. C'est qu'il faut se garder de poser un domaine extérieur au réel, sorte d'arrière-monde spéculatif, résultat d'une fabulation; à la place, on doit concevoir un monde virtuel «défini comme une stricte partie de l'objet réel »(Deleuze, Différence 269). On ne sera donc pas étonné d'apprendre que Deleuze critique vertement la notion de possible : il y voit l'expression claire d'une extériorité par rapport au réel.

2) Mais il est une seconde raison qui amène le Français à prendre ses distances vis-à-vis du possible : «le possible et le virtuel se distinguent [...] parce que l'un renvoie à la forme d'identité dans le concept, tandis que l'autre désigne une multiplicité pure dans l'Idée, qui exclut radicalement l'identique comme condition préalable» (273). Anne Sauvagnargues l'exprime en ces termes : la possibilité correspond au «réel, moins sa réalité » (Sauvagnargues, Philosophie 126). Une relation d'identité existe ainsi au plan représentationnel entre le possible et le réel : l'un et l'autre ont un contenu identique, bien que le premier, au contraire du second, n'ait aucun 
ancrage dans le monde effectif. De cette relation d'identité, Deleuze estime qu'elle a un inconvénient majeur, celui de faire oublier l'existence d'une «multiplicité pure». Cette multiplicité est plus fondamentale que toute forme d'identité, car elle représente la condition transcendantale de l'individu. Nous expliquerons en quel sens plus loin. Pour l'instant, bornonsnous à dire que Deleuze a le souci de balayer l'idée classique de possible au motif qu' elle ne fait pas droit, au contraire du virtuel, qui exclut le principe d'identité, au rôle structurel de la multiplicité pure.

3) Quel est le troisième argument qui amène l'auteur à rejeter le concept de possible? De manière presque pédagogique, Deleuze l'introduit lui-même par un marqueur de relation: «[e]nfin, dans la mesure où le possible se propose à la "réalisation", il est [...] conçu comme l'image du réel, et le réel, comme la ressemblance du possible. C'est pourquoi l'on comprend si peu ce que l'existence ajoute au concept, en doublant le semblable par le semblable» (Deleuze, Différence 273). L'argument esquissé ici repose sur une conviction : «tout n'est pas donné, ni donnable» (Zourabichvili 89). Thème d'inspiration bergsonienne (cf. Bergson, Évolution créatrice, entre autres $5 s q$.), que Deleuze reprend à son compte : l'existence du temps révèle le caractère inachevé de l'univers, c'est-à-dire le fait que s'y poursuit sans trêve une «création d'imprévisible nouveauté » (Bergson, «Possible et réel » 99). Mais ce travail de création est recouvert par l'idée de possible. Tel qu'on le pense le plus souvent, ou tel, du moins, que le pense Deleuze, le possible n'est autre qu'une idéalité préexistant au réel et à l'image du réel luimême. Prenons le processus de délibération. Lorsqu'on le décrit en assumant l'idée de possible, on dit qu'il s'agit, pour celui qui délibère, de jeter son dévolu sur une option possible préexistante et dont l'actualisation n'ajoute rien d'essentiellement nouveau à l'option, toute alternative étant déjà à l'image du réel. Deleuze s'insurge contre cette description. Pour lui, 
puisque tout n'est pas donné et qu'il s'opère dans le monde une création continue; puisque, par ailleurs, l'idée de possible ne rend pas manifeste cet élan de création (parce qu'on la dit à l'image du réel sans tenir compte de ce que l'actualisation ajoute au concept), il convient d'abandonner cette idée au profit du virtuel.

De ces diverses considérations, ainsi que de celles qui précèdent, découle sans détour la conclusion à laquelle il fallait en venir : un parallèle peut être tracé entre Heidegger et Deleuze. En effet, leurs philosophies respectives ont ceci de commun qu'elles se montrent très critiques vis-à-vis des acceptions classiques du possible et que, pour justifier ces critiques, elles s'attèlent toutes deux à tailler en pièces ces différents sens — traditionnels ou courants. Il est vrai que les deux auteurs ne s'en prennent pas exactement aux mêmes sens de l'idée de possible; mais l'un et l'autre se rejoignent par le mécontentement qu'ils affichent vis-à-vis de la tradition, et Deleuze se rapproche de Heidegger en ce qu'il vitupère trois préjugés massifs qu'on peut rattacher, sinon à l'ensemble des conceptions classiques du possible, du moins à certaines d'entre elles ${ }^{8}$.

\section{Un moment positif : la possibilité existentiale et le concept de virtuel}

Si l'on sait à présent en quoi ne consistent pas les positions de Heidegger et Deleuze sur l'ordre modal, il reste encore à établir un point : comment se déterminent ces positions et quelles sont leurs conséquences propres? Répondre à cette question apparaît d'autant plus pressant qu'il deviendra possible, ce faisant, de dégager deux recoupements majeurs ${ }^{9}$. Afin de définir la possibilité existentiale, Heidegger développe le concept de «comprendre »: «[1]e comprendre est l'être existential du pouvoir-être propre du Dasein lui-même [...] [II] a en lui-même la structure existentiale que nous appelons le projet. [...] Le caractère de projet du comprendre 
constitue l'être-au-monde du point de vue de l'ouverture de son Là comme Là d'un pouvoir-être. Le projet est la constitution existentiale d'être de l'espace de jeu du pouvoir-être factice» (Heidegger, Être et temps 127 sq.). Plus que des affirmations de passage, ces remarques sont révélatrices. Elles expliquent que le comprendre renvoie à la situation d'être-au-monde du Dasein et que, dans cette situation, se dévoile la structure du projet. Par projet, il faut entendre l'ouverture fondamentale en fonction de laquelle le Dasein peut réaliser des possibilités. «Le Dasein se comprend toujours déjà et toujours encore, aussi longtemps qu'il est, à partir de possibilités » (128). Heidegger est donc autorisé à dire que la possibilité prend le pas sur l'effectivité (49), dans la mesure où le Dasein dépend des possibilités pour se projeter, s'exprimer, s'affirmer de quelque façon dans le monde.

Mais le dessein de Heidegger n'est pas tant d'attirer l'attention sur les «possibilités déterminées » que sur «l'espace de jeu» sur lequel elles font fond. En cela consiste donc, précisément, la possibilité existentiale du Dasein : loin de se rapporter à un réseau de possibilités déterminées, actualisables ou tout au moins imaginables, elle correspond au possible pur ou, pour mieux dire, à la structure originaire en vertu de laquelle il y a possibilité. L'insistance de Heidegger sur ce possible pur est constante. Dans Être et temps (127), pour ne mentionner qu'un endroit, Heidegger soutient qu'au moment où se réalise une possibilité, au moment où elle acquiert l'actualité, les possibilités non réalisées s'avèrent mises de côté. Or, toujours d'après lui, il convient moins de s'intéresser à la réalisation de possibilités données que de reconduire l'esprit vers le domaine du possible pur, d'où émergent ces possibilités. Tout se passe à vrai dire comme si Heidegger cherchait à opérer une conversion du regard : plutôt que d'inciter le Dasein à se préoccuper des possibilités $\mathrm{X}, \mathrm{Y}, \mathrm{Z}$, il l'enjoint d'avoir en tête, d'abord et avant tout, qu'un 
pouvoir-être structurel marque son expérience, et donc que l'ordre des choses n'est jamais réifiable, arrêté, figé.

En maints endroits dans son œuvre, l'auteur précise le sens de ce pouvoir-être et de l'idée de possibilisation (Ermöglichung). Ses analyses sont bien connues. Heidegger identifie la mort à «la possibilité la plus propre, absolue, indépassable »(201), c'est-à-dire à une possibilité soustraite à toute effectivité et capable de révéler le sens de l'excédent constant. Il affirme par ailleurs que l'expérience de l'angoisse nous met en contact avec le néant (« Métaphysique » 59), de sorte qu'elle nous reconduit vers un domaine extérieur à l'étant, où loge le pouvoir-être (Être et temps 156). Aussi Heidegger précise-t-il le sens du rapport entre Dasein et possibilisation. «Le Néant est la condition qui rend possible la révélation de l'existant comme tel pour la réalitéhumaine. [...] C'est dans l'être de l'existant que se produit le néantir du Néant» («Métaphysique » 63). Deux idées cardinales, l’une implicite, l'autre explicite, se profilent ici. Premièrement, le néant est la condition de possibilité de l'être, car c'est dans l'horizon du néant que tout vient à se manifester. Deuxièmement, la constitution transcendantale de l'être n'est pas due à l'individu comme tel. Car le Dasein n'est pas à l'origine de la possibilisation de l'être. Il se trouve impliqué dans ce processus, sans doute, puisqu'il lui revient de configurer ses possibilités et de s'engager dans le monde (Être et temps 130). Mais contrairement à Husserl, Heidegger ne fait pas de l'individu l'agent de la constitution transcendantale, qu'il considère plutôt comme un patient, puisqu'il ne constitue pas lui-même son propre pouvoir-être.

À preuve, Heidegger s'attarde sur ce qu'il appelle les «existentiaux »: il les définit comme les «structures d'existence »(55) du Dasein, mais comme des structures telles qu'elles ne sont pas forgées par le Dasein lui-même. Si bien que la possibilité existentiale — qui a un caractère structurel - ne peut être constituée sur un mode subjectif. La Lettre sur l'humanisme 
fournit des indications similaires : elle pointe en direction d'une atténuation de l'activité du sujet. Heidegger y revient sur une affirmation d'Être et temps, selon laquelle « [1]'essence de l'être-là réside dans son existence » («Das “Wesen” des Daseins liegt in seiner Existenz ») :

il ne s'agit pas là d'une opposition entre existentia et essentia [cf. Etre et temps 54], car ces deux déterminations métaphysiques de l'Être en général, et à bien plus forte raison leur rapport, ne sont pas encore en question. La phrase contient moins encore un énoncé général sur l'être-là [...] Bien plutôt veut-elle dire que l'homme déploie son essence de telle sorte qu'il est le « là », c'est-à-dire l'éclaircie de l'Être. Cet « être » du là, et lui seul, comporte le trait fondamental de l'ek-sistence, c'est-à-dire de l'in-stance extatique dans la vérité de l'Être. L'essence extatique de l'homme repose dans l'ek-sistence, qui reste distincte de l'existentia pensée d'un point de vue métaphysique. («Lettre sur l'humanisme » $81 s q$.)

Chez Platon, l'essence précède l'existence; chez Sartre, le contraire prévaut. Heidegger récupèret-il l'opposition classique entre existence et essence? On le constate dans l'extrait qui précède : il n'en est rien. L'Allemand substitue aux termes de cette opposition un concept inédit, l'eksistence, de manière à déplacer le problème et initier un «nouveau commencement» de la pensée. Son ambition est donc de se détacher à la fois de Platon et de Sartre, et de recentrer la question autour de la vérité de l'être, le Dasein n'étant pas libre, au contraire de ce que dit Sartre, de forger sa propre essence, mais capable, néanmoins, de tenir compte de cette essence et de configurer des possibilités à partir de son pouvoir-être — ce que ne dit pas Platon ${ }^{10}$.

Récapitulons. La pensée heideggérienne a pour objectif de revisiter le problème classique du possible. Pour ce faire, elle introduit le concept de possibilité existentiale dont le caractère structurel indique que le pouvoir-être conditionne l'expérience du sujet. S'il est loisible de caractériser la philosophie de Heidegger comme transcendantale, c'est donc pour autant qu'on voie en elle autre chose qu'un idéalisme. Ce point de vue sur le possible s'accorde-t-il avec la pensée de Deleuze? Abstraction faite de leurs critiques des sens courants du possible, on pourrait 
croire que les philosophies heideggérienne et deleuzienne sont fort éloignées, dans la mesure où l'une transporte le possible vers le plan existential, tandis que l'autre le congédie au profit d'une nouvelle notion. Mais elles convergent à bien des égards. Soucieux de rendre compte du sujet, Deleuze développe une théorie de la multiplicité et de la différence. Qu'est-ce donc qu'un individu? Comment en expliquer l'avènement? Telles sont les questions auxquelles il tente de répondre en puisant dans l'œuvre de Gilbert Simondon. Pour Simondon (L'individu et sa genèse physico-biologique), il importe de comprendre l'individu à partir de sa genèse. À la question de savoir ce qu'est le sujet, il substitue ainsi l'interrogation suivante: comment se fait-il? Si Deleuze abonde dans le sens de Simondon et conçoit que le processus d'individuation est plus essentiel que l'individu lui-même, c'est par respect pour deux principes implicites : d'une part, l'individu ne préexiste pas à la multiplicité ${ }^{11}$, toute force individuelle étant constituée dans un rapport de forces multiples, au cours d'un processus d'individuation (cf. entre autres Deleuze, Différence 71 et Logique 125); de l'autre, tout individu est appelé à changer au cours du temps, changement auquel ne pourrait rendre justice une pensée qui ne met pas au premier plan l'individuation ${ }^{12}$. On entrevoit là les bases d'une théorie du virtuel. Quel en est le contenu exact?

Deleuze distingue deux pôles qui sont «mutuellement exclusifs tout en étant joints » (Boundas 19) : l'actuel (l'individu) et le virtuel (les singularités). Dans Logique du sens, il avance qu'on ne peut comprendre l'individuation qu'en posant l'existence d'un champ préindividuel de constitution, plan matériel où se meuvent des «singularités anonymes et nomades, impersonnelles » (Deleuze, Logique 125) ${ }^{13}$. La matière virtuelle de ce plan peut être caractérisée par opposition aux trois acceptions du possible (relevées plus haut) que rejette Deleuze : (1) elle n'est pas extérieure au réel, mais ancrée dans l'objectivation; (2) au lieu de supposer des individus stables, pré-donnés et antérieurs à toute relation, elle implique une multiplicité 
intensive où tout demeure indéterminé ( $c f$. notamment «Actuel et virtuel » $179 s q$.); (3) loin de correspondre à une idéalité qui serait à l'image du réel, elle se trouve en voie d'actualisation et porte l'empreinte du devenir. Toujours dans le sillage de Simondon, Deleuze décrit l'individuation en énonçant une hypothèse : si la matière virtuelle tend à s'actualiser pour former un individu ${ }^{14}$, c'est qu'elle se situe dans un système « métastable » où surgit un problème ${ }^{15}$.

Anne Sauvagnargues le résume comme suit : «La structuration objective mais métastable d'un champ, que Simondon nomme le "problématique", contient une différence, un disparate, un déséquilibre potentiel qu'il ne s'agit pas d'éliminer, mais de résoudre par disparation, sur un mode créateur, en produisant une dimension nouvelle qui ne préexistait pas au problème » (Sauvagnargues 134 sq.; cf. Deleuze, Logique 125). Ainsi apparaissent donc l'individu et tout corps individué : en réponse à un déséquilibre de potentiels qui suscite un problème, les singularités virtuelles se collisionnent et produisent un sujet, réponse extensive à un problème intensif. Cette thèse sur l'individuation se noue à une conception de la différence en soi. Alors que la tradition n'avait conçu que des différences relatives, à savoir des différences entre individus constitués, Deleuze veut atteindre «l'en-soi de la différence [...], c'est-à-dire [...] la différence avec soi qui rapporte le différent au différent par soi-même » (Différence 157 sq.). Au cœur de cette différence, grouille un champ de singularités multiples, hétérogènes, prégnantes, qui défient l'identité fixe parce qu'en voie d'actualisation. La conclusion à en tirer est double.

1) Tout d'abord, de même que Heidegger attire l'attention sur le possible pur, plus fondamental que toute forme d'actualité, de même Deleuze fait dépendre le sujet actuel d'une instance extérieure à lui : les singularités. L'accent de Deleuze porte en effet sur le pôle du virtuel, qui n'est rien de moins que le moteur de l'individu. Mais alors, faut-il admettre, les pensées heideggérienne et deleuzienne ont un point en commun : toutes deux nous reconduisent 
de l'actualité vers un domaine plus essentiel, ici le possible pur, là le virtuel, toutes deux se prêtent sur un sujet un mutuel appui. Le corollaire en est que l'expérience ne se réduit pas aux possibilités réalisées ni davantage à celles dont on nous fait croire qu'elles sont les seules réalisables. On nous objectera peut-être que Deleuze attribue au virtuel une certaine forme de réalité, alors que Heidegger distingue le possible pur de l'ordre du réel (Différence 157). Mais cela n'interrogera en rien l'entente de fond qui existe entre eux, car Deleuze fait du virtuel une condition de possibilité et un devenir, ce qui indique que le réel est toujours en train de se faire, capable de se transformer. Pour sa part, Heidegger rappelle que le réel est débordé par le possible pur, en vertu de quoi il demeure lui-même en phase avec la nouveauté, paré aux transformations, ouvert au changement.

2) Outre sa réflexion sur le virtuel, Deleuze développe une thèse transcendantale. Bien qu'il adresse de vifs reproches à la tradition transcendantale (cf. par ex. Logique 129), qui négligerait le fait que les catégories de pensée sont produites (sur cette idée précise, $c f$. Richard et aussi Deleuze, Empirisme et subjectivité), il s'en autorise lui-même. À ce titre, par exemple, il dit du virtuel qu'il constitue un « champ transcendantal » (Deleuze, Logique 133) ou encore que les singularités pré-individuelles, concept qu'il fait sien, «sont les vrais événements transcendantaux »(125). Une similitude de plus existe donc entre Heidegger et Deleuze : tandis que le premier développe une pensée transcendantale qui atténue le rôle actif du sujet, le second minimise ce rôle en établissant l'existence d'un champ virtuel ${ }^{16}$.

L'aspect transcendantal n'est pas secondaire chez les deux philosophes. L'Allemand rattache au pouvoir-être une dimension structurelle et, quand il ne le rapproche pas de l'être même ${ }^{17}$, notion la plus générale qui soit, il le place au-dessus. Telle est du moins la lecture qu'en fait Deleuze : «dans ses derniers écrits Heidegger ne parle même plus de métaphysique ni de 
dépassement de la métaphysique, puisque l'être à son tour doit être dépassé, au profit d'un Pouvoir-Être » («Précurseur méconnu »118) ${ }^{18}$. De son côté, la philosophie deleuzienne pose d'entrée de jeu l'existence d'un champ transcendantal pré-individuel, ce qui amène Éric Alliez — fait peu banal — à lui conférer l'épithète de philosophie virtuelle ${ }^{19}$. Non seulement existe-t-il ainsi un lien patent entre Heidegger et Deleuze, mais de plus, ce lien se laisse déduire d'une zone essentielle de leurs pensées.

\section{Cap sur la pratique : une complémentarité possible?}

Les sections qui précèdent ont établi divers recoupements entre Heidegger et Deleuze, qui ont tous trait au versant théorique de leurs pensées. Or, qu'en est-il du versant pratique? En matière éthico-politique, comment concevoir la relation entre Heidegger et Deleuze? Ce serait une lapalissade de dire que Deleuze a une grande sensibilité politique. Avec des ouvrages tels que L'Anti-CEdipe et Mille Plateaux, il a jeté les bases, de concert avec d'autres ${ }^{20}$, d'un projet politique dont s'inspirèrent plusieurs ( $c f$. notamment Hardt et Negri, Goulimari et MoulardLeonard). Moins politisé, Heidegger a quant à lui souffert d'avoir soutenu le national-socialisme, et sans doute est-ce en partie par souci de prudence qu'il limita ses commentaires politiques dès la fin des années 1930 .

L'engagement politique de Heidegger a fait couler beaucoup d'encre ( $c f$. par ex. Farias, et la réponse de Fédier, Heidegger). Puisqu'on sait aujourd'hui qu'il ne prenait pas les Juifs en aversion (Fédier, «Revenir ») et qu'il lui était impossible, en 1933, de prévoir l'ampleur des atrocités que commettrait le régime nazi, nous ne reviendrons pas sur cet engagement. Bien plutôt, nous tenterons de montrer que la pensée deleuzienne est de nature à compléter la pensée 
heideggérienne. À l'exception notable de quelques articles et de diverses allocutions ( $c f$. Heidegger, Écrits politiques), dont le «Discours de rectorat » où est lancé un appel à l'autoaffirmation du peuple allemand, Heidegger demeure assez réticent à l'idée de formuler des directives pratiques. Cette distance vis-à-vis de l'action se manifeste à plusieurs reprises. Ainsi, dans la Lettre sur l'humanisme, Heidegger affirme que sa pensée «n'est ni théorique ni pratique »(119), mais antérieure à cette distinction. Dans L'histoire de l'être, il soutient sans vergogne qu' « aucune révolution n'est assez révolutionnaire », comme quoi tout projet politique représente un faux pas pour peu qu'il soit fondé sur l'étant, plutôt que sur la vérité de l'être et le pouvoir-être qui s'y imbrique ${ }^{21}$. Interrogé par Der Spiegel, Heidegger signale du reste que «travail et défense sont comme toute activité humaine fondés dans un savoir et éclairés par lui » («Spiegel », 246) et, à la question de savoir si la philosophie peut influencer le monde d'une façon ou d'une autre, il répond «[qu']une autre pensée pourrait avoir une influence médiate, mais aucune directe d'une façon qui ferait dire que la pensée "cause" un changement de l'état du monde $\gg(261)$.

Que Heidegger fasse l'impasse sur le volet pratique et qu'il n'en ait somme toute cure, cela est donc manifeste, mais d'autant plus déplorable qu'il dit lui-même du Dasein qu'il s'engage toujours déjà dans le monde (Être et temps 128), ce qui exige en principe une réflexion approfondie en matière pratique. Cette réflexion approfondie, on ne la retrouve pas chez Heidegger. Tout au plus y repère-t-on une théorie de l'authenticité. Jean-Édouard André en a fait la démonstration (André 251 sq.) : cette théorie a des conséquences sur l'agir social du Dasein. Car le Dasein doit se méfier des lieux communs et faire l'effort de configurer ses possibilités d'existence. Il lui incombe de prendre acte du fait qu'un pouvoir-être possibilise son expérience et qu'en ce sens, premièrement, une part de liberté lui revient et, deuxièmement, aucune décision 
humaine n'est définitive ${ }^{22}$. Heidegger nous informe donc que les circonstances actuelles sont débordées par d'autres manières d'exister. N'en demeure pas moins que cette réflexion est assez peu restrictive. En effet, si elle excelle à nous rappeler que toute construction humaine pourrait être renversée (le capitalisme, le régime représentatif, etc.), elle est empreinte d'un certain flou : elle ne fournit guère d'indications sur les actions politiques à privilégier. Pour le dire autrement, à insister tant sur le possible pur plutôt que sur les possibilités existentielles, Heidegger omet de se demander si la réalisation de certains possibles constitue une action plus digne, plus souhaitable, voire tout à fait primordiale ${ }^{23}$.

Pour définir les contours d'une action juste, certains iront peut-être puiser chez Marx des directives pratiques. Ils trouveront alors des raisons de lutter contre le capitalisme et le travail aliéné, qui génèrent des inégalités et cantonnent l'homme dans la production de marchandises vénales. Ici, en complétant la pensée heideggérienne par la pensée marxienne, ils permettraient en quelque sorte à Marx de prendre sa revanche sur Heidegger. Faut-il préciser que celui-ci reprochait à son prédécesseur d'avoir adopté un préjugé fautif, celui selon lequel l'actualité a préséance sur le possible ${ }^{24}$ ? Mais il n'y a pas que la doctrine marxienne qui soit susceptible de compléter le corpus heideggérien: les réflexions de Deleuze en sont également capables. Deleuze et Heidegger pointent tous deux en direction d'un horizon semblable, le possible pur et le virtuel. En cela, ils semblent déjà plus près de s'entendre que Marx et le phénoménologue.

En quoi le système deleuzien est-il à même de renchérir sur celui de Heidegger? Deleuze s'intéresse à la pratique en tenant compte du primat à accorder à ce qui transcende l'actuel, primat déjà mis en lumière par Heidegger lui-même. Il fait le départ entre deux types de segments : les tendances «molaires» et les tendances «moléculaires»(Deleuze et Guattari, Plateaux 260). Les premières sont à rattacher à une «macropolitique »; les secondes, à une 
«micropolitique ${ }^{25}$. Étant constituées de «segmentarités dures», les tendances molaires travaillent à la centralisation des forces et à la fixation du devenir. Elles trouvent leur expression dans l'État, la bureaucratie et les revendications identitaires. À l'opposé, les tendances moléculaires impliquent des «lignes de fuite» et des «zones de déterritorialisation»: elles affolent l'identité en « coulant » entre les segments durs (263). En raison du mouvement qui leur est propre, on peut associer les tendances molaires au pôle de l'actuel et les tendances moléculaires à celui du virtuel. Personne ne contestera que Deleuze cherche avant tout à faire valoir le second pôle. Dans un chapitre intitulé «1730 - Devenir-intense, devenir-animal, devenir-imperceptible... »(284-380), il nous convie ainsi à une entreprise de désubjectivation dont la fin première n'est pas de devenir ceci ou cela, de faire révolution en substituant au pouvoir en place un nouveau pouvoir ${ }^{26}$, mais de devenir..., encore et encore, par une activation des forces disponibles.

Ce projet micropolitique se justifie d'au moins deux manières. (1) D'une part, en luttant contre la fixité, on rend justice aux singularités pré-individuelles, sans lesquelles nul individu ne pourrait advenir, mais que recouvrent trop souvent les institutions molaires (l'État, les forces de l'ordre, etc.), avec pour résultat de faire oublier qu'aucune actualité n'est définitive. (2) D'autre part, la micropolitique permet d'initier une véritable praxis de la communauté, où l'individu n'est plus pensé comme entité stable, mais toujours agencé avec ce qu'il n'est pas, sans cesse enrichi par les rencontres. Deleuze est catégorique sur ce point: mener à bien un projet de désubjectivation exige de faire preuve à la fois d'«ascèse » et de «sobriété » (Plateaux, notamment 13 et 342). D'ascèse, puisqu'on doit apprendre à renoncer à soi et à faire corps avec le multiple; de sobriété, car sans elle, on poussera l'expérimentation trop loin et on risquera de devenir des «loques », dont l'impuissance rend impossible le devenir de demain (185-204). À 
ces grands traits se résume donc l'entreprise de désubjectivation micropolitique. Si Deleuze en chante les louanges, il ne manque pas de s'intéresser, par ailleurs, à l'aspect macropolitique :

Toute société, mais aussi tout individu, sont [...] traversés par les deux segmentarités à la fois : l'une molaire et l'autre moléculaire. [...] Bref, tout est politique, mais toute politique est à la fois macropolitique et micropolitique. Soit des ensembles du type perception, ou sentiment : leur organisation molaire, leur segmentarité dure, n'empêche pas tout un monde de micro-percepts inconscients, d'affects inconscients, segmentations fines, qui ne saisissent ou n'éprouvent pas les mêmes choses, qui se distribuent autrement, qui opèrent autrement. (260)

L'extrait est sans équivoque. Les deux segmentarités politiques, molaire et moléculaire, vont nécessairement de pair. C'est en effet toujours en vertu d'une certaine poussée moléculaire que l'ordre molaire peut apparaitre, et toute poussée moléculaire, parallèlement, converge vers une direction donnée, c'est-à-dire qu'elle tend vers l'identité (aspiration molaire). Deleuze a beau mettre l'accent sur la micropolitique, il fait donc également cas des tendances molaires ${ }^{27}$. Évoquons l'exemple des luttes féministes. Traditionnellement, ces combats sont identitaires: lorsqu'on ne réclame pas un traitement égal et des droits égaux pour les hommes et les femmes, tout en posant une certaine différence entre les sexes (Simone de Beauvoir), on remarque une différence essentielle entre eux et on milite pour faire reconnaitre cette différence (Luce Irigaray). Deleuze transporte le combat ailleurs. Pour lui, la lutte est à mener avant tout à travers le prisme du devenir : il importe aux femmes d'engendrer un «devenir-femme », d'entraîner autrui dans ce devenir et de conduire tout un chacun — les femmes et autrui — dans l'imperceptibilité, l'indiscernabilité, l'impersonnalité (340-343).

$\mathrm{Au}$ contraire de ce qu'on pourrait croire, toutefois, ce projet demeure compatible avec les revendications identitaires. Dépourvues de certains droits et de certains traitements, les femmes n'auront pas l'espace pour s'exprimer en société, elles ne seront pas en mesure de s'affirmer 
publiquement. Forcées de céder le passage aux hommes, elles n'auront ni voix au chapitre ni la capacité d'embarquer autrui dans le devenir qui leur est propre. Il semble dès lors légitime pour elles de lutter pour une reconnaissance, fût-ce afin de rendre possible un devenir-femme désubjectivisant ( $c f$. également Deleuze et Parnet 176, où il est question d'un « droit au désir »). En d'autres termes, de l'avis même de Deleuze, pour autant que les revendications identitaires s'accompagnent d'un «mouvement plus profond»(Deleuze et Guattari, Plateaux 589) qui célèbre le devenir et ravale la constance, elles ont tout à fait droit de cité : «lutte des femmes pour le vote, pour l'avortement, pour l'emploi », mais aussi, hors des cadres féministes, «lutte des régions pour l'autonomie; lutte du tiers monde; lutte des masses et des minorités opprimées » (588; $c f$. Antonioli 95).

Qu'en conclure? Le projet politique de Deleuze est à l'image de sa réflexion sur l'actuel et le virtuel : s'il se centre plus volontiers sur l'un des deux pôles, le micropolitique, il ne néglige pas pour autant son pendant, le macropolitique. Deleuze appelle donc de ses vœux une action politique concrète et il fournit en détail ce que ne fournissait pas Heidegger lui-même. En fait, le Français détermine une zone d'action laissée indéterminée par l'Allemand : il précise quelles possibilités actualiser, comment les actualiser et, du même coup, il laisse entrevoir une manière possible de compléter la pensée heideggérienne d'un point de vue pratique.

\section{Conclusion}

Vu son contact renouvelé avec les écrits de Heidegger, Deleuze devait s'y sentir en terrains connus, mais assurément pas chez soi. Non seulement les commentaires abondent qui remarquent une distance nette entre les deux, mais aussi, Deleuze se disait lui-même fort éloigné 
de Heidegger. N'empêche que les deux philosophes se rejoignent sur plusieurs plans : d'une part, ils mènent un combat acharné contre les conceptions classiques du possible; d'autre part, ils braquent leurs armes dans le même sens lorsqu'ils démontent une idée courante, celle selon laquelle la priorité va à l'actuel plutôt qu'à un autre horizon, le possible pur ou le virtuel; enfin, ils développent une philosophie qui fait place au transcendantal. On forcerait cependant le trait en rapprochant Heidegger et Deleuze sous l'ensemble des points de vue. Alors que l'Allemand tourne le dos au versant pratique, le Français développe une pensée à caractère politique ( $c f$. Negri). Un certain fossé sépare donc bel et bien Heidegger et Deleuze; mais qui dit fossé, dit aussi possibilité d'ériger un pont, et c'est sur la construction de ce pont que nous avons travaillé ici, en tentant de dégager une complémentarité possible ${ }^{28}$.

Non pas que l'Allemand se serait à coup sûr reconnu dans le Français ou que celui-ci aurait aimé être couplé à celui-là — il le qualifiait parfois de «druide nazi » (cf. Goetz). Mais la pensée deleuzienne fournit effectivement les moyens de préciser ce qui ne l'est pas chez Heidegger. Là où Heidegger se tait, c'est-à-dire dans le domaine pratique, Deleuze s'exprime : il nous invite à une micropolitique doublée de revendications molaires. Nul ne saurait nier que Deleuze contracte lui-même une dette envers Heidegger. En effet, c'est précisément Heidegger qui fut le premier à redonner ses droits à un horizon irréductible à l'actuel. Prendre la pleine mesure du rapport entre Heidegger et Deleuze exige sans doute, dès lors, de mettre en évidence la contribution propre de Heidegger; car si le Français va plus loin que l'Allemand en ce qu'il précise quelles possibilités actualiser et comment les actualiser, il ne crée pas de toutes pièces ses idées politiques, il les développe en tenant compte du primat à accorder à ce qui transcende l'actuel — réquisit tout heideggérien ${ }^{29}$. 


\section{Notes}

${ }^{1}$ Il va de soi que Deleuze ne fait pas pour autant l'éloge de la technique, encore qu'il tienne bel et bien par moments à s'approprier le langage de la technique et à lui attribuer un sens mélioratif. Aussi parle-t-il de «machines étatiques », dont le sens est péjoratif.

2 Il faut noter que Deleuze ponctue son œuvre de références furtives à Heidegger, qui ne sont pas toutes résolument critiques ( $c f$. par ex. Deleuze et Guattari, Philosophie 43).

3 Pour une analyse de la signification de la mort (qui s'intrique au ne-pas-encore du Dasein, et sur laquelle nous reviendrons) telle qu'elle apparaît dans le versant métaphysique de la pensée heideggérienne, $c f$. Brisart.

4 Rappelons au passage l'existence de deux articles fondamentaux sur Bergson, écrits par Deleuze : «Bergson. 1859-1941 » et «La conception de la différence chez Bergson ». Sur les raisons qui motivent Bergson à mettre en question l'idée classique de possible, $c f$. notamment Bergson, « Possible et réel ».

5 Pour un examen de deux de ces arguments, qu'on retrouve aussi chez Bergson, le troisième n'apparaissant de manière explicite que chez Deleuze, $c f$. Fradet, "Auscultation ». Pour une étude du rapport entre Heidegger, Leibniz et Bergson, $c f$. Perrin.

6 Pour une analyse de ce présupposé, que Deleuze semble emprunter à Bergson, $c f$. Fradet, «Bergson, Heidegger ». Dans le présent article, nous suggérons que Bergson met en question à titre premier et principal le concept de possibilité logique. Il n'est pourtant pas exclu qu'il s'en prenne aussi au concept de possibilité réelle. Sans doute, la philosophie bergsonienne arrime sa conception du possible au réel, puisqu'elle attribue au virtuel une certaine forme de réalité. Mais il serait réducteur d'affirmer que cette philosophie endosse en tout point le concept de possibilité réelle, car Bergson déplace et repense dans une large mesure le problème même du possible (ainsi que plusieurs concepts traditionnels) en proposant l'idée de virtuel.

7 Sur le caractère objectif des singularités virtuelles elles-mêmes, $c f$. notamment Deleuze, Logique 127. Soulignons à ce titre que Deleuze, qui enracine le virtuel dans un «passé pur », rejette l'alternative métaphysique portant à croire que les singularités ne sont pas distinguables : «ou bien un fond indifférencié, sans-fond, non-être informe, abîme sans différences et sans propriétés — ou bien un Être souverainement individué, une Forme fortement personnalisée » (129).

8 Il est incontestable, par exemple, que le troisième argument de Deleuze l'autorise à critiquer la notion de possibilité logique, également interrogée par Heidegger, qui se lance à la quête d'une possibilité plus fondamentale. 
${ }^{9}$ Que Deleuze se rapproche de Heidegger sous différents angles, comme on le verra d'ici peu, cela peut s'expliquer en partie par le fait qu'il dit lui-même s'en inspirer sur la question de la différence (Deleuze, Différence 89-91). À ce propos, $c f$. Dronsfield 151 sq. et Bell $114 s q$.

${ }^{10} \mathrm{Au}$ sujet de l'essence et de l'humanisme chez Heidegger, $c f$. Marquet. Pour un prolongement d'analyse sur le rapport entre Heidegger et l'existentialisme, $c f$. Beaufret 18-32.

${ }^{11}$ N'en déplaise à Alain Badiou (Deleuze) qui fait de Deleuze le penseur de l'Un, «l'essentiel de l'univocité n'est pas que l'Être se dise en un seul et même sens. C'est qu'il se dise, en un seul et même sens, de toutes ses différences individuantes ou modalités intrinsèques. L'Être est le même pour toutes ces modalités, mais ces modalités ne sont pas les mêmes » (Deleuze, Différence 53).

12 Pour une affirmation du caractère instable de l'individu, $c f$. ., parmi bien d'autres passages, Deleuze, Différence 130 et 139. Pour une mise en évidence de cette idée chez Simondon, cf. L'individuation 26. Certains remarqueront sans doute que ce raisonnement n'est autre qu'une généralisation hâtive ou abusive. Car ce n'est pas parce qu'on repère des changements, à certains moments et chez certains individus, que tout individu à tout moment demeure disposé à changer. Aussi est-il possible de croire que certains changements s'expliquent, non pas par l'aspect temporel des choses, mais par des lois stables (ou relativement stables) que relève la science en mettant l'accent plus sur l'individu que sur l'individuation.

${ }^{13}$ Ce plan n'est pas un «arrière-monde inaccessible », et c'est en partie pourquoi Deleuze le situe à la « surface » - le sens étant en quelque sorte superficiel.

14 À des fins de clarification terminologique, Anne Sauvagnargues («Deleuze avec Bergson » 158) mentionne ceci : "Le virtuel, réel mais idéal, est différentié (avec un "t") dans son contenu sans qu'il ait besoin de s'actualiser; lorsqu'il s'actualise, il se différencie (avec un "c"). » Par ailleurs, pour une analyse complémentaire de Simondon qui transcende la lecture qu'en fait Deleuze, $c f$. Barthélémy.

15 Iain Macdonald nous a rapporté ce fait : s'il est un désaccord entre Heidegger et Deleuze, il tient sans doute davantage au matérialisme de ce dernier, position dont s'écarte plutôt Heidegger. Toutefois, ce désaccord ne doit pas masquer les rapprochements possibles entre les deux auteurs.

${ }^{16}$ La diminution du rôle actif de l'individu prend plusieurs formes chez Heidegger. Décrivant l'expérience quotidienne du Dasein dans Être et temps, il en parle comme d'un être-aumonde préoccupé par les étants qui l'entourent, étants dont le sens ne s'établit jamais dans l'indépendance et l'isolement, mais toujours en vertu de la «relationnalité » et du «renvoi » (Être et temps 73 sq.) par lesquels un étant se distingue des autres. On comprendra donc que pour Heidegger, aucun étant discret, individu ou objet, ne se suffit à lui-même. Puisque cette idée n'engage qu'implicitement une mise en question de l'activité du sujet, nous avons préféré nous concentrer ici sur d'autres passages, plus explicites, pour définir le sens de cette critique chez Heidegger. 
17 Ce rapprochement peut être observé, entre autres, lorsque Heidegger rapatrie l'être et le possible autour d'un concept commun, l'Il y a : $c f$. «Temps et être » 198 sq. et $202 s q$.

18 Il faut mentionner que Deleuze fait reposer son commentaire sur la conférence «Temps et être ». Sur les exigences de dépassement des problèmes classiques et de transformation de la pensée, $c f$. aussi Heidegger, « Fin de la philosophie ».

19 Même les commentaires rédigés par Deleuze semblent à vrai dire portés par une foi dans le pré-individuel. Commentant Proust, Deleuze suggère ainsi que l'acte de penser est créé, causé par une violence extérieure (Proust 189 sq.).

20 On peut penser notamment à Foucault, pour l'éthique de la désubjectivation. Pour prendre connaissance d'autres penseurs, occidentaux ou chinois, de l'impersonnalité, $c f$. en particulier Bordeleau.

21 Sur le désaveu heideggérien du politique au profit d'une pensée de «l'archi-politique », cf. Lacoue-Labarthe 161.

22 Sur le rapport entre le Dasein et le «On», qui conduit vers l'inauthenticité, $c f$. Heidegger, Etre et temps 114 sq.

23 Quoique l'accent de Heidegger porte davantage sur la théorie que sur la pratique, il n'hésite pas à dire, rejoignant en cela Deleuze, que «[p]enser, ce n'est pas ne rien faire; la pensée est elle-même en soi l'action dans son dialogue avec le monde entendu comme destin » (Heidegger, «Spiegel » 265, nous soulignons).

${ }^{24}$ Heidegger devient plus reconnaissant envers Marx dans un passage précis : $c f$. « Lettre sur l'humanisme » $98 \mathrm{sq}$.

${ }^{25}$ Mis à part une dimension politique, la pensée deleuzienne possède aussi un aspect proprement éthique, où l'appel est lancé à l'élargissement de la puissance d'agir et à l'accroissement de la joie. Si nous nous attardons ici en particulier à la dimension politique, c'est que celle-ci, premièrement, s'articule à l'aspect éthique de la pensée de Deleuze, et, deuxièmement, que certaines implications éthiques des philosophies heideggérienne et deleuzienne ont déjà été étudiées par Adkins. Notons-le au passage : pour clarifier la genèse de l'éthique de Deleuze, il serait nécessaire de considérer entre autres ces travaux : Nietzsche et la philosophie, Nietzsche et Spinoza et le problème de l'expression. Afin de prendre la pleine mesure de l'éthique deleuzienne, il faudrait également considérer la manière dont Deleuze s'insère lui-même dans ses commentaires et met en œuvre une pratique, en même temps qu'il relève l'élément pratique des pensées qu'il commente ( $c f$. Lemieux).

26 Par cette critique de la révolution, projet qu'écarte Deleuze au profit d'un jeu de variation continue, l'auteur se rapproche quelque peu de Heidegger qui se méfie lui-même des révolutions politiques. 
27 L'accent mis par Deleuze sur la micropolitique amène différents auteurs, dont Philippe Mengue, à interroger la portée pratique de cette lutte pour les peuples du tiers-monde. En tenant compte du pendant molaire du projet deleuzo-guattarien, qu'évoque aussi Mengue (76), on peut cependant atténuer cette critique, tout en reconnaissant que les auteurs insistent (peut-être à l'excès) sur la micropolitique. Pour une réflexion sur l'emploi à faire de la micropolitique, qui recouvre quelquefois d'autres types de combats possibles, plus ou moins fondés ou adéquats selon les circonstances, $c f$. par ailleurs Fradet, Anonymous Photographs.

28 Pour un autre exemple de rapprochement entre Heidegger et un auteur qui, en apparence, s'en éloigne à certains égards, $c f$. Macdonald et Ziarek.

29 Il me faut remercier ici Iain Macdonald et René Lemieux pour leurs judicieux commentaires, ainsi que les organismes qui m'ont offert leur appui financier lors des recherches ayant mené à la préparation de cet article : le CRSH, le FQRSC et la Fondation Desjardins.

\section{Textes cités}

ADKINS, Brent, Death and Desire in Hegel, Heidegger, and Deleuze, Édimbourg, Edinburgh University Press, 2008.

AlLIEZ, Éric, Deleuze. Philosophie virtuelle, Paris, Synthélabo, 1996.

ANDRÉ, Jean-Édouard, Heidegger et la politique. L'épreuve de la liberté, Paris, L'Harmattan, 2006.

Antonioli, Manola, «La machination politique de Deleuze et Guattari », in A. Beaulieu (dir.), Gilles Deleuze, héritage philosophique, Paris, P.U.F., 2005.

BADIOU, Alain, Deleuze. «La clameur de l'être », Paris, Hachette, 1997.

BARTHÉLÉMY, Jean-Hugues, «Penser après Simondon et par-delà Deleuze », Cahiers Simondon, $\mathrm{n}^{\circ} 2,2010$, p. 129-146.

BEAUFRET, Jean, De l'existentialisme à Heidegger, Paris, Vrin, 1986.

BeAulieu, Alain, Gilles Deleuze et la phénoménologie, Sils Maria, Vrin, 2004.

—, «The Status of Animality in Deleuze's Thought», Journal for Critical Animal Studies, vol. $9, \mathrm{n}^{\circ} 1-2,2011$, p. 69-88. 
Bell, Jeffrey A., Philosophy at the Edge of Chaos. Gilles Deleuze and the Philosophy of Difference, Toronto, University of Toronto Press, 2006.

Bergson, Henri, «Le possible et le réel », in Bergson, La pensée et le mouvant, Paris, P.U.F., 2008, p. 99-116.

—, L'évolution créatrice, Paris, P.U.F., 2007.

BoRDELEAU, Érik, «[E]scape. Anonymat et politique à l'ère de la mobilisation globale: passages chinois pour la communauté qui vient », thèse de doctorat, Département de littérature comparée, Université de Montréal, 2009.

Boundas, Constantin V., «Les stratégies différentielles dans la pensée deleuzienne », in A. Beaulieu (dir.), Gilles Deleuze, héritage philosophique, Paris, P.U.F., 2005.

BRISART, Robert, «La métaphysique de Heidegger », in F. Volpi et al. (dir.) Heidegger et l'idée de la phénoménologie, Dordrecht, Kluwer Academic Publishers, 1988.

Brown, Lori, «Becoming-Animal in the Flesh. Expanding the Ethical Reach of Deleuze and Guattari's Tenth Plateau », PhonEx, vol. 2, n² 2, 2007, p. 260-278.

Buchanan, Brett, Onto-Ethologies. The Animal Environments of Uexküll, Heidegger, MerleauPonty, and Deleuze, Albany, Suny Press, 2008.

Deleuze, Gilles, «L'actuel et le virtuel », in G. Deleuze et C. Parnet, Dialogues, Paris, Flammarion, 1996.

—, «Bergson. 1859-1941», in M. Merleau-Ponty (dir.) Les philosophes célèbres, Paris, Éd. d'art Lucien Mazenod, 1956, p. 292-299.

—, Le bergsonisme, Paris, P.U.F., 2004.

—, «La conception de la différence chez Bergson », in Deleuze, Études bergsoniennes, Paris, Albin Michel, 1956.

—, Différence et répétition, Paris, P.U.F., 2005.

—, Empirisme et subjectivité, Paris, P.U.F., 2003.

—, Logique du sens, Paris, Minuit, 1969.

—, «Un précurseur méconnu de Heidegger, Alfred Jarry », in Deleuze, Critique et clinique, Paris, Minuit, 1993.

_, Proust et les signes, Paris, P.U.F., 1971. 
Deleuze, Gilles et Félix GuatTari, L'Anti-CEdipe, Paris, Minuit, 1972.

_, Mille plateaux, Paris, Minuit, 1980.

—, Qu'est-ce que la philosophie?, Paris, Minuit, 2005.

Deleuze, Gilles et Claire PARnet, Dialogues, Paris, Flammarion, 1996.

DRONSFIELD, Jonathan, «Between Deleuze and Heidegger There Never is any Difference », in D. Pettigrew et F. Raffoul (dir.), French Interpretations of Heidegger. An Exceptional Reception, Albany, Suny Press, 2008.

FARIAS, Victor, Heidegger et le nazisme, trad. M. Benarroch et J.-B. Grassert, Paris, Verdier, 1987.

FÉDIER, François, Heidegger. Anatomie d'un scandale, Paris, Robert Laffont, 1988.

—, « Revenir à plus de décence », in Heidegger, Écrits politiques par Martin Heidegger, 19331966, trad. F. Fédier, Paris, Gallimard, 1995, p. 9-96.

FoucAult, Michel, «L'Anti-CEdipe. Une introduction à la vie non fasciste », trad. F. DurandBogaert, Magazine littéraire, $\mathrm{n}^{\circ} 257$, sept. 1988, disponible sur Internet : http://multitudes.samizdat.net/L-Anti-Oedipe-Une-introduction-a.

Fradet, Pierre-Alexandre, Anonymous Photographs of the Past. A Petition Against Death?, trad. T. Widom et P.-A. Fradet, Santa Fe, CSF Publishing, 2012.

- «Auscultation d'un cour battant. L'intuition, la durée et la critique du possible chez Bergson », Laval théologique et philosophique, vol. 67, n³ 3, 2011, p. 531-552.

-, «Bergson, Heidegger et la question du possible. Le renversement d'une conception classique », Ithaque, vol. 8, 2011, p. 97-117.

GoETz, Benoît, «L'araignée, le lézard et la tique. Deleuze et Heidegger lecteurs de Uexküll », Le Portique, $\mathrm{n}^{\circ}$ 20, 2007, disponible sur Internet : http://leportique.revues.org/index1364.html.

Goulimari, Pelagia, «A Minoritarian Feminism? Things to do with Deleuze and Guattari », Hypatia, vol. 14, n 2, 1999.

HARDT, Michael et Antonio NEGRI, Empire, trad. D.-A. Canal, Paris, Exils, 2000.

HeGEL, Encyclopédie des sciences philosophiques, trad. B. Bourgeois, Paris, Vrin, 1986. 
—, Science de la logique, trad. S. Jankélévitch, Paris, Aubier, 1949.

HeIDEGGER, Martin, Écrits politiques par Martin Heidegger, 1933-1966, trad. F. Fédier, Paris, Gallimard, 1995.

—, Être et temps, trad. E. Martineau, Authentica (éd. hors-commerce), 1985.

—, «La fin de la philosophie et la tâche de la pensée », in Heidegger, Questions III et IV, trad. J. Beaufret et F. Fédier, Paris, Gallimard, 1976.

—, «Lettre sur l'humanisme », in Heidegger, Questions III et IV, trad. R. Munier, Paris, Gallimard, 1976.

—, «Martin Heidegger interrogé par Der Spiegel », trad. J. Launay, in Heidegger, Écrits politiques par Martin Heidegger, 1933-1966, trad. F. Fédier, Paris, Gallimard, 1995, p. 239-272.

—, «Qu'est-ce que la métaphysique? », in Heidegger, Questions I et II, trad. H. Corbin, Paris, Gallimard, 1968.

—, «La question de la technique », in Heidegger, Essais et conférences, trad. A. Préau, Paris, Gallimard, 1958, p. 9-48.

—, « Temps et être », in Heidegger, Questions III et IV, trad. F. Fédier, Paris, Gallimard, 1976.

Lacoue-Labarthe, Philippe, Heidegger. La politique du poème, Paris, Galilée, 2002.

LEMIEUX, René, «Hume et Bergson, une pratique de la méthode chez Deleuze. Réflexions pour une éthique de la lecture », Symposium, vol. 13, n² 2, 2009, p. 68-96.

MacdonAlD, Iain et Krzysztof ZIAREK (dir.), Adorno and Heidegger, Stanford, Stanford University Press, 2008.

MARQUeT, Jean-François, «Une étrange sorte d'humanisme », in B. Pinchard (dir.), Heidegger et la question de l'humanisme. Faits, concepts, débats, Paris, P.U.F., 2005.

MEmon, Arsalan, «Merleau-Ponty, Deleuze, and the Question Singular. What Marks the Difference Between Humans and Animals? », Auslegung. A Journal of Philosophy, vol. 28, $\mathrm{n}^{\circ} 2$, 2006, p. 19-35.

Mengue, Philippe, Gilles Deleuze ou le système du multiple, Paris, Kimé, 1994.

Moulard-Leonard, Valentine, «Revolutionary Becomings. Negritude's Anti-Humanist Humanism », Human Studies, vol. 28, n 3, 2005, p. 231-249. 
Negri, Antonio, «Qu'est-ce que la philosophie, selon Deleuze et Guattari », trad. G. Donnard, Multitudes, vol. 8, 1991,

http://webcache.googleusercontent.com/search?hl=en\&gbv=2\&q=cache:5F5MVUZuqa4J:http:// multitudes.samizdat.net/Qu-est-ce-que-la-philosophie-selon+deleuze+heidegger\&ct=clnk.

PERrin, Christophe, «Heidegger et Leibniz : de la simplicité du rien », Klêsis, n 7, 2008, p. 4356.

PIERON, Julien, «Monadologie et/ou constructivisme? Heidegger, Deleuze, Uexküll », Bulletin d'analyse phénoménologique, vol. 6, $\mathrm{n}^{\circ} 2,2010$.

RICHARD, Christophe, «L'empirisme transcendantal », in J.-M. Vaysse (dir.), Technique, monde, individuation : Heidegger, Simondon, Deleuze, Hildesheim, Olms, 2006.

Sauvagnargues, Anne, «Deleuze avec Bergson. Le cours de 1960 sur L'évolution créatrice », in F. Worms (dir.), Annales bergsoniennes II. Bergson, Deleuze, la phénoménologie, Paris, P.U.F., 2004.

—, La philosophie de Deleuze, Paris, P.U.F., 2004.

SERGEANT, Philippe, Nietzsche, de l'humour à l'éternel retour, Paris, éd. de la Différence, 2010.

SIMONDON, Gilbert, L'individu et sa genèse physico-biologique, Paris, P.U.F., 1964.

_, L'individuation à la lumière des notions de forme et d'information, Grenoble, Millon, 2005.

VAYSSE, Jean-Marie, «Nietzsche entre Heidegger et Deleuze », in J.-M. Vaysse (dir.), Technique, monde, individuation : Heidegger, Simondon, Deleuze, Hildesheim, Olms, 2006.

WinCHESTER, James J., Nietzsche's Aesthetic Turn. Reading Nietzsche after Heidegger, Deleuze, Derrida, Albany, Suny Press, 1994.

WITT, Charlotte, Unity, Identity, and Explanation in Aristotle's Metaphysics, Oxford, Oxford University Press, 1994.

ZourABICHVILI, François, Le vocabulaire de Deleuze, Paris, Ellipses, 2003. 